\title{
Simulations of polarized dust emission
}

\author{
V.-M. Pelkonen* and M. Juvela \\ Department of Astronomy, University of Helsinki, Tähtitorninmäki, P.O.Box 14,SF-00014 \\ University of Helsinki, Finland \\ E-mail: vpelkone@astro.helsinki.fi, mjuvela@astro.helsinki.fi

\section{P. Padoan} \\ Department of Physics, University of California, San Diego, CASS/UCSD 0424, 9500 Gilman \\ Drive, La Jolla, CA 92093-0424 \\ E-mail: ppadoan@ucsd.edu
}

\begin{abstract}
Our aim is to study the polarization of thermal dust emission to see if the alignment of grain by radiative torques could explain the observed relation between the degree of polarization and the intensity in dense cores. Predictions are made for polarimetry observations with the Planck satellite. Our results are based on model clouds derived from MHD simulations of magnetized turbulent flows, while the continuum radiative transfer problem is solved with Monte Carlo methods in order to estimate the three-dimensional distribution of dust emission and the radiation field strength affecting the grain alignment. The influence of grain alignment efficiency is examined in the calculated polarization maps. We are able to reproduce the P/I-relation with the grain alignment by radiative torques. The decrease in intrinsic polarization and total emission means that sub-mm polarimetry carries only little information about the magnetic fields in dense cores with high visual extinction. The interpretation of the observations will be further complicated by the unknown magnetic field geometry and the fact that what is observed as individual cores may, in fact, be a superposition of several density enhancements. According to our calculations, Planck will be able to map dust polarization reliably when $A_{\mathrm{V}}$ exceeds $\sim 1^{\mathrm{m}}$.
\end{abstract}

$C M B$ and Physics of the Early Universe

Ischia, Italy

April, 20-22 2006

\footnotetext{
* Speaker.
} 


\section{Introduction}

The radiative torque mechanism is a strong candidate as the primary mechanism of grain alignment inside molecular clouds. Cho \& Lazarian [1] showed that even deep inside GMCs large grains can be aligned by radiative torques and reproduced the observed drop in the P/I-relation using a simple spherical model cloud. Padoan et al. [2] used numerical MHD simulations of self-gravitating cores to model a molecular cloud, with the simplifying assumption that the dust temperature was uniform. They were able to reproduce the observed drop by introducing a sharp cut-off at $A_{\mathrm{V}}=3$. Our work is also based on 3D MHD models [3], but we refine the analysis method used to predict the polarized dust emission. Radiative transfer calculations [ [ 4 [5] are used to estimate the actual 3D distribution of the dust emission and to estimate the radiative torque efficiency of Cho \& Lazarian [1]. This way, we can test the hypothesis that reduced polarization of dense cores could be due to the non-alignment of smaller grains. We also make some predictions for polarimetry observation using the Planck satellite.

\section{Polarization}

\subsection{Rayleigh Polarization Reduction Factor}

The Rayleigh polarization reduction factor $R$ is a measure of imperfect alignment of the dust grains with respect to the magnetic field [6] [7]. In the case of radiative torques, small grains are not aligned while the larger ones are. Cho and Lazarian [1] give a fitting formula for the minimum aligned grain size: $a_{\mathrm{alg}}=\left(\log n_{\mathrm{H}}\right)^{3}\left(A_{\mathrm{V}}+5\right) / 2800 \mu \mathrm{m}$, where $n_{\mathrm{H}}$ is the hydrogen density and $A_{\mathrm{V}}$ is the effective visual extinction, i.e., the extinction of the external interstellar radiation field that is illuminating the cloud.

The polarization reduction factor is

$$
R=\frac{\int_{a_{\mathrm{alg}}}^{a_{\max }} C_{\mathrm{ran}} n(a) d a}{\int_{a_{\min }}^{a_{\max }} C_{\mathrm{ran}} n(a) d a},
$$

where $C_{\mathrm{ran}}$ is the average polarization cross section of a randomly oriented grain, $n(a)$ the grain number density, $a$ the grain size, $a_{\min }$ the minimum size of the grains, $a_{\max }$ the maximum size, and $a_{\text {alg }}$ the minimum aligned grain size. Figure 1 shows the polarization reduction factor for different densities and maximum grain sizes.

\subsection{Polarized Thermal Dust Emission}

The polarized thermal dust emission is calculated following the formalism in Fiege \& Pudritz [10]. Self-absorption and scattering can be neglected at submillimeter wavelengths.

The Stokes Q and U components are proportional to the integrals

$$
\begin{aligned}
q & =\int \alpha f \cos 2 \psi \cos ^{2} \gamma d s, \\
u & =\int \alpha f \sin 2 \psi \cos ^{2} \gamma d s,
\end{aligned}
$$

where $\alpha$ is a coefficient of the particle properties to be defined later, $f$ a weighting function related to the intensity of the local dust emission, $\psi$ the angle between the projection of $\mathbf{B}$ on the plane 


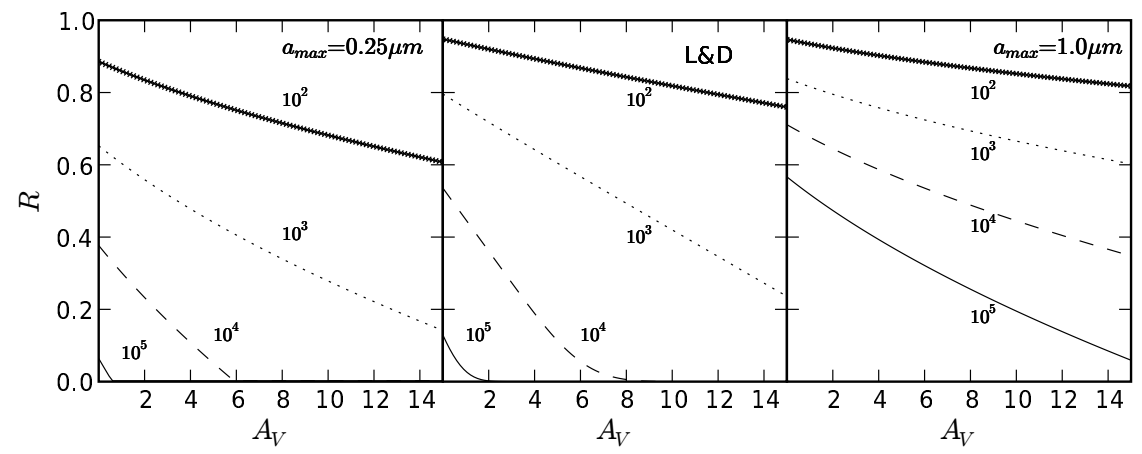

Figure 1: Polarization reduction factor $R$ as a function of $A_{\mathrm{V}}$ using Weingartner \& Draine [8] dust model. The curves correspond to different densities that are given in the figure in units of $\mathrm{cm}^{3}$. The frames correspond to different values of the maximum grain size, $a_{\max }$. In the first and the third frame the grain size distributions are pure power laws, $n(a) \sim a^{-3.5}$, while in the middle frame the size distribution is taken from Li \& Draine [9].

of the sky and the north, and $\gamma$ the angle between the local $\mathbf{B}$ vector and the plane of the sky. The polarization angle $\chi$ is given by $\tan 2 \chi=u / q$ and the degree of polarization $P$ is

$$
P=\frac{\sqrt{q^{2}+u^{2}}}{\Sigma-\Sigma_{2}}
$$

with $\Sigma=\int f d s$ and $\Sigma_{2}=\frac{1}{2} \int \alpha f\left(\cos ^{2} \gamma-\frac{2}{3}\right) d s$.

The coefficient $\alpha$ is defined as $\alpha=R F C_{\mathrm{pol}} / C_{\mathrm{ran}}$, where $R$ is the Rayleigh polarization reduction factor, $F$ is the polarization reduction factor due to the turbulent component of the magnetic field (in this study, $F=1$ ) and $C_{\mathrm{pol}}$ is the grain polarization cross section. For more details, see Lee \& Draine [才]. In our study, we use both uniform $R=1$ as well as $R$ which varies according to the local efficiency of the radiative torque, as explained in Section 2.1. The other parameters are chosen to scale $\alpha$ so that the maximum polarization is about $15 \%$ when $R=1$.

\section{Results}

\subsection{P/I-relation}

We scale our self-gravitating model cloud to the size $L=6 \mathrm{pc}$ and mean density $n=640 \mathrm{~cm}^{-3}$. This results in an optically thick cloud with average visual extinction $\left\langle A_{\mathrm{V}}>\sim 6.3^{\mathrm{m}}\right.$. In the case of variable $R$, the polarization degree at low column densities varies between $\sim 4$ and $10 \%$ while, at the centre of the cores, the degree drops to a few percent or, in some cases, even below one percent. In other words, our physical model is capable of explaining the general observed behaviour. At the same time, the individual cores show considerable variation, depending on the actual configuration of the magnetic field. One apparent problem is that from observations it may be difficult to discern real and possibly gravitationally bound cores from projection effects.

\subsection{Predictions for the Planck satellite}

For the predictions, we want to study if the polarized signal is detectable in regions of low column density. Thus, we scale the column densities of the cloud down by a factor of ten, resulting 

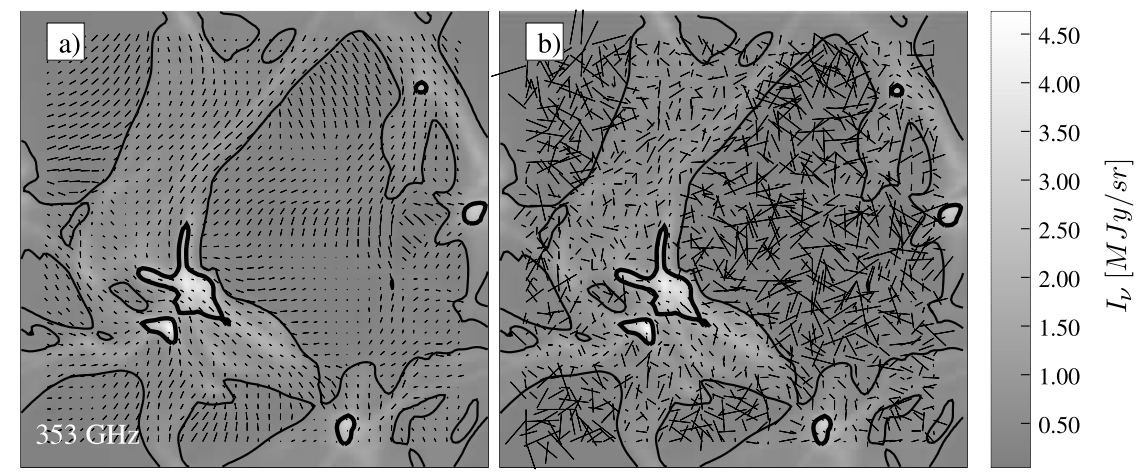

Figure 2: Simulated polarization map at $353 \mathrm{GHz}$ without noise (left frame) and with noise corresponding to observations with the Planck satellite (right hand frame). Without noise the maximum polarization degree is $8 \%$. In the noisy map, long vectors are cut down to a maximum value of $25 \%$. One pixel corresponds to the Planck resolution of 5 arcmin. Polarization vectors are drawn for every third pixel along both axes. The countours are drawn for $A_{\mathrm{V}}$ values of 0.5 and 2.5 magnitudes.

in a model with average extinction $\left\langle A_{\mathrm{V}}>\sim 0.6^{\mathrm{m}}\right.$. Simulations are done with variable $R$ and with noise appropriate to the Planck instruments at $353 \mathrm{GHz}$. Figure 2 1 shows the computed polarization map with contours corresponding to $A_{\mathrm{V}}$ values of 0.5 and 2.5 magnitudes. The polarization vectors follow, with some scatter, the magnetic fields even along filaments with $A_{\mathrm{V}} \sim 1^{\mathrm{m}}$ or above. The sensitivity can be increased by reducing the spatial resolution, leading to better correlation between true and 'observed' field morphology. Thus, we conclude that at the resolution of $\sim 10^{\prime}$ Planck will be able to make reliable maps of dust polarization in regime where $A_{\mathrm{V}}$ exceeds $\sim 1^{\mathrm{m}}$.

\section{References}

[1] J. Cho and A. Lazarian, Grain Alignment by Radiation in Dark Clouds and Cores, ApJ, 631 (2005), 361

[2] P. Padoan, A. Goodman, B.T. Draine, et al., Theoretical Models of Polarized Dust Emission from Protostellar Cores, ApJ, $\mathbf{5 5 9}$ (2001), 1005

[3] M. Juvela, P. Padoan, \& A. Nordlund, Cooling Rates of Molecular Clouds Based on Numerical Magnetohydrodynamic Turbulence and Non-LTE Radiative Transfer, ApJ, 563 (2001), 853

[4] M. Juvela and P. Padoan, Dust emission from inhomogeneous interstellar clouds: Radiative transfer in $3 D$ with transiently heated particles, $A \& A, 397$ (2003), 201

[5] M. Juvela, Efficient Monte Carlo methods for continuum radiative transfer, A\&A, 440 (2005), 531

[6] J.M. Greenberg, Nebulae and Interstellar Matter, Vol. 7, Univ. Chicago Press, Chicago 1968

[7] H. Lee and B.T. Draine, Infrared extinction and polarization due to partially aligned spheroidal grains - Models for the dust toward the BN object, ApJ, 290 (1985), 211 (1985)

[8] J.C. Weingartner and B.T. Draine, Dust Grain-Size Distributions and Extinction in the Milky Way, Large Magellanic Cloud, and Small Magellanic Cloud, ApJ, 548 (2001), 296

[9] A. Li and B.T. Draine, Infrared Emission from Interstellar Dust. II. The Diffuse Interstellar Medium, ApJ, 554 (2001), 778

[10] J.D. Fiege and R.E. Pudritz, Polarized Submillimeter Emission from Filamentary Molecular Clouds, ApJ, 544 (2000), 830 